\title{
GENERALIZED EIGENFUNCTIONS OF THE LAPLACE OPERATOR AND WEIGHTED AVERAGE PROPERTY
}

ANIL KUMAR BOSE

I. Introduction. In the previous two papers, [1] and [2], we were interested in characterizing the class $S(w, R)$ of real-valued functions $u$, defined in a given region (open, connected set) $R$ of the $n$-dimensional Euclidean space $E_{n}$ which satisfy the Weighted Average Property (W.A.P.):

$$
u(P)=\int_{B(P, r)} u \cdot w d \rho / \int_{B(P, r)} w d \rho, \quad P \in R,
$$

where $B(P, r)$ denotes any ball with the point $P=P\left(x_{1}, x_{2}, \cdots, x_{n}\right)$ for its center and radius $r$ whose closure lies in $R$; $d \rho$ stands for the usual Lebesgue measure of $B$ and $w$ is a weight function (W.F.) defined in $R$ (i.e., $w$ is nonnegative and locally summable in $R$ ).

It was proved in paper [1] that " $S(w, R)$ is always a subspace of the solution space of the second-order linear elliptic homogeneous differential equation:

$$
w \Delta u+2 \sum_{i=1}^{n} u_{x_{i}} w_{x_{i}}=0
$$

in $R$, where $u$ is the Laplacian of $u$, provided $w \in C^{1}(R)$." Furthermore, " $S(w, R)$ is the solution space of the equation (2) if the W.F. $w$ is an eigenfunction of the Laplace operator. That is, if $w$ is a solution of an equation of the form:

$$
\Delta w+\lambda w=0
$$

in $R$, where $\lambda$ is some real constant." In the latter case " $S(w, R)$ is infinite dimensional."

The present paper is an extension of these results. In this paper we want to prove that " $S(w, R)$ is always a subspace of the (common) solution space of a system of equations of the form:

$$
\Delta^{k} w \cdot \Delta u+2 \sum_{i=1}^{n} u_{x_{i}}\left(\Delta^{k} w\right)_{x_{i}}=0, \quad k=0,1,2,3, \cdots,
$$

where $\Delta^{k} w$ is the $k$ th iteration of the Laplacian of $w\left(\Delta^{0} w\right.$ is interpreted as $w)$, provided the weight function $w$ is sufficiently differentiable."

Presented to Society, January 27, 1967; received by the editors December 12, 1966. 
Furthermore, " $S(w, R)$ is precisely the solution space of the system of equations (4), provided the weight function $w$ is a generalized eigenfunction of the Laplace operator or more generally a weight function satisfying an equation of the form:

$$
\Delta^{m} w=a_{0} w+a_{1} \Delta w+\cdots+a_{m-1} \Delta^{m-1} w,
$$

in the region $R$, where $m$ is a positive integer and the $a_{i}$ 's are real constants."

In paper [2] it was proved that "in $E_{2}, S(w, R)$ is infinite dimensional if and only if $w$ is an eigenfunction of the Laplace operator; otherwise, $S(w, R)$ is finite dimensional and $1 \leqq \operatorname{dim} S(w, R) \leqq 2$." It was also claimed there that in $E_{n}, n>2$, " $S(w, R)$ is infinite dimensional if and only if $w$ is an eigenfunction of the Laplace operator; otherwise, $S(w, R)$ is finite dimensional and $1 \leqq \operatorname{dim} S(w, R) \leqq 2 n-1$." In a footnote of [2] it was mentioned that the above statement is, possibly, not true in $E_{n}, n>2$. The main result of this paper clarifies this point completely. Indeed, it will be shown in \$III of this paper that in $E_{n}, n>2, S(w, R)$ could be infinite dimensional even if the weight function $w$ is not an eigenfunction of the Laplace operator.

II. TheOREM 1. Let $R$ be a region in $E_{n}$ and w be a W. F. defined in $R$.

(i) If $w \in C^{2 m+1}(R), m$ being a nonnegative integer, then $S(w, R)$ is a subspace of the solution space of the system of equations:

$$
\Delta^{k} w \cdot \Delta u+2 \sum_{i=1}^{n} u_{x_{i}}\left(\Delta^{k} w\right)_{x_{i}}=0, \quad \text { in } R, k=0,1,2, \cdots, m .
$$

(ii) If $\Delta^{k} w$ is defined and is in $C^{1}(R)$ for each positive integer $k$, then $S(w, R)$ is a subspace of the solution space of the system of equations:

$$
\Delta^{k} w \cdot \Delta u+2 \sum_{i=1}^{n} u_{x_{i}}\left(\Delta^{k} w\right)_{x_{i}}=0 \quad \text { in } R, k=0,1,2,3, \cdots, \infty
$$

Proof. Part (i). Let $u \in S(w, R)$. Then by Theorem 2 and Theorem 4 of $[1], u \in C^{2 m+2}(R)$ and

$$
w \Delta w+2 \sum_{i=1}^{n} u_{x_{i}} w_{x_{i}}=0 \text { in } R .
$$

Also by Theorem 3 of [1], we get the circumferential mean-value property

$$
\int_{S(P, r)} u w d o=u(P) \int_{S(P r)} w d \sigma
$$


for each $S(P, r)$-the boundary of $B(P, r)$-which, together with its interior $B(P, r)$, lies in $R$. As proved in Theorem 4 of [1], we get immediately from (8), by differentiating with respect to $r$, the meanvalue relation

$$
u(P) \int_{S(P, r)} \frac{\partial w}{\partial n} d \sigma=\int_{S(P, r)} \frac{\partial u w}{\partial n} d \sigma
$$

for each $S(P, r)$ which, together with its interior $B(P, r)$, lies in $R$, where $\partial / \partial n$ refers to the derivative in the direction of the outward drawn normal to the surface $S(P, r)$. Now, using Green's formula and relations (7) and (9), we get

$$
\begin{aligned}
\int_{B(P, r)}\left(w \Delta u+2 \sum_{i=1}^{n} u_{x_{i}} w_{x_{i}}+u \Delta w\right) d \rho & =\int_{B(P, r)} \Delta(u w) d \rho \\
=\int_{S(P, r)} \frac{\partial u w}{\partial n} d \sigma & =u(P) \int_{S(P, r)} \frac{\partial w}{\partial n} d \sigma
\end{aligned}
$$

or

$$
\int_{B(P, r)} u \Delta w d \rho=u(P) \int_{B(P, r)} \Delta w d \rho,
$$

for each $B(P, r)$ whose closure lies in $R$. It is now clear that we can apply the entire reasoning of Theorem 4 of [1] over again to the averaging property (10) and get

$$
\Delta w \cdot \Delta u+2 \sum_{i=1}^{n} u_{x_{i}}(\Delta w)_{x_{i}}=0 \quad \text { in } R .
$$

Since $\Delta^{k} w$ is defined and is in class $C^{1}(R)$ for $k=0,1,2, \cdots, m$, repeating the argument a finite number of times, we see that $u$ must satisfy the system of equations:

$$
\Delta^{k_{w}} \cdot \Delta u+2 \sum_{i=1}^{n} u_{x_{i}}\left(\Delta^{k_{w}}\right)_{x_{i}}=0, \quad k=0,1,2, \cdots, m \text { in } R .
$$

This proves Part (i).

Part (ii). Let $u \in S(w, R)$. Since $\Delta^{k} w$ is defined and is in class $C^{1}(R)$ for all $k=0,1,2,3, \cdots$, the above reasoning together with mathematical induction implies that $u$ must be a common solution of the system of equations:

$$
\Delta^{k} w \cdot \Delta u+2 \sum_{i=1}^{n} u_{x_{i}}\left(\Delta^{k_{w}}\right)_{x_{i}}=0
$$


in $R, \quad k=0,1,2,3, \cdots, \infty$. This proves Part (ii).

Theorem 2. Defined in a region $R$ of $E_{n}$, let w be a W. F. belonging to class $C^{2 m}(R)$ and be a solution of the differential equation

$$
\Delta^{m} w=a_{0} w+a_{1} \Delta w+\cdots+a_{m-1} \Delta^{m-1} w
$$

in $R$, where $m$ is a positive integer and the $a_{i}$ 's are real constants. A realvalued function $u$ is in $S(w, R)$ if and only if $u$ is in $C^{2}(R)$ and is a common solution of the system of equations:

$$
\Delta^{k} w \cdot \Delta u+2 \sum_{i=1}^{n} u_{x_{i}}\left(\Delta^{k} w\right)_{x_{i}}=0, \quad k=0,1,2, \cdots, m-1, \quad \text { in } R .
$$

Proof. Let $u \in S(w, R)$. By Theorem 2 of $[1], u \in C^{2 m+1}(R)$. Since $w \in C^{2 m}(R)$, the right-hand side of $(12)$ is in $C^{2}(R)$. Hence, $\Delta^{m+1} w$ is defined in $R$ and is a linear combination of $w, \Delta w, \Delta^{2} w, \cdots, \Delta^{m-1} w$ in $R$. Applying mathematical induction, it is easy to see that $\Delta^{m+p} w$ is defined in $R$ and $\Delta^{m+p} w$ is a linear combination of $w, \Delta w, \Delta^{2} w, \cdots$, $\Delta^{m-1} w$ in $R$ for each positive integer $p$. This means that $\Delta^{k} w$ is defined in $R$ and is a linear combination of $w, \Delta w, \Delta^{2} w, \cdots, \Delta^{m-1} w$ in $R$ for each positive integer $k$. Hence, by Part (ii) of Theorem 1, it follows that $u$ is a common solution of the system of equations

$$
\Delta^{k} w \cdot \Delta u+2 \sum_{i=1}^{n} u_{x_{i}}\left(\Delta^{k} w\right)_{x_{i}}=0
$$

in $R, k=0,1,2,3, \cdots, \infty$. Hence, $u$ is a common solution of system (13) of equations.

Conversely, suppose that $u \in C^{2}(R)$ and is a common solution of the system of equations (13) in $R$. Since $\Delta^{k} w$ is defined in $R$ and is a linear combination of $w, \Delta w, \Delta^{2} w, \cdots, \Delta^{m-1} w$ for each positive integer $k$, it follows that every member of the system (14) of equations can be expressed as a linear combination of the $m$ equations of the system (13). That is, $u$ is also a common solution of the system (14) of equations in $R$. Since $\Delta(u w)$ is defined in $R$ and $u$ is a solution of

$$
w \Delta u+2 \sum_{i=1}^{n} u_{x_{i}} w_{x_{i}}=0 \quad \text { in } R
$$

we have

$$
\Delta(u w)=w \Delta u+2 \sum_{i=1}^{n} u_{x_{i}} w_{x_{i}}+u \Delta w=u \Delta w \quad \text { in } R .
$$


Now, assuming that $\Delta^{k}(u w)$ is defined and $\Delta^{k}(u w)=u \Delta^{k} w$ in $R$ for some positive integer $k$ and using the fact that $u$ is a solution of the system (14) of equations in $R$, we see that $\Delta^{k+1}(u w)$ is defined in $R$ and

$$
\Delta^{k+1}(u w)=\Delta^{k} w \cdot \Delta u+2 \sum_{i=1}^{n} u_{x_{i}}\left(\Delta^{k} w\right)_{x_{i}}+u \Delta^{k+1} w=u \Delta^{k+1} w \quad \text { in } R .
$$

Hence, by mathematical induction $\Delta^{k}(u w)$ is defined in $R$ and

$$
\Delta^{k}(u w)=u \cdot \Delta^{k} w
$$

in $R$ for each positive integer $k$. Putting $k=m$ and using (12), we see that

$$
\Delta^{m}(u w)=u \Delta^{m} w=a_{0}(u w)+a_{1} \Delta(u w)+\cdots+a_{m-1} \Delta^{m-1}(u w) .
$$

Therefore, $u w$ is also a solution of equation (12) in $R$. Let $B(P, r)$ be any ball which, together with its boundary $S(P, r)$, lies in $R$. As proved in [3, pp. 286-289].

$$
\begin{aligned}
\frac{1}{\Omega_{r}} \int_{S(P, r)} w d \sigma= & \Gamma(n / 2) \sum_{\nu=0}^{m+k-1}(r / 2)^{2 \nu} \frac{\Delta^{\nu} w(P)}{\nu ! \Gamma(\nu+n / 2)} \\
& +\int_{B(P, r)} v_{m+k}(\beta) \Delta^{m+k} w d \rho
\end{aligned}
$$

for all positive integers $k$, where $\Omega_{r}$ is the surface area of $S(P, r)$ and the sequence of functions $v_{m}(\beta)$ are given by the recursion system

$$
\begin{aligned}
v_{v+1}(\beta) & =\frac{1}{(n-2) \beta^{n-2}} \int_{\beta}^{r} \alpha v_{\nu}(\alpha)\left(\alpha^{n-2}-\beta^{n-2}\right) d \alpha, \\
v_{0}(\beta) & =\left(1 /(n-2) \Omega_{1}\right)\left(1 / \beta^{n-2}-1 / r^{n-2}\right),
\end{aligned}
$$

$\Omega_{1}$ being the surface area of the unit sphere in $n$-dimensional space and $\beta$ being the distance of a point in $B(P, r)$ from the center $P$. [In two dimensions the recursion formula $(17)$ is given by

$$
\begin{aligned}
v_{\nu+1}(\beta) & =\int_{\beta}^{r} \alpha v_{\nu}(\alpha) \log \alpha / \beta d \alpha, \\
v_{0}(\beta) & =(1 / 2 \pi) \log r / \beta .]
\end{aligned}
$$

Since $\Delta^{m} w=a_{0} w+a_{1} \Delta w+\cdots+a_{m-1} \Delta^{m-1} w$, we have $\Delta^{m+k} w=a_{0}^{(k)} w+a_{1}^{(k)} \Delta w+\cdots+a_{m-1}^{(k)} \Delta^{m-1} w, \quad k=1,2,3, \cdots, \infty$, where the sequences of constants $\left\{a_{i}^{(\boldsymbol{k})}\right\}, i=0,1,2, \cdots, m-1$, are defined recursively by 


$$
\begin{aligned}
& a_{0}^{(k)}=a_{m-1}^{(k-1)} a_{0}, \\
& a_{1}^{(k)}=a_{0}^{(k-1)}+a_{m-1}^{(k-1)} a_{1}, \cdots, a_{m-1}^{(k)}=a_{m-2}^{(k-1)}+a_{m-1}^{(k-1)} a_{m-1},
\end{aligned}
$$

$a_{i}^{(0)}$ being interpreted as $a_{i}, i=0,1, \cdots, m-1$.

Let $c / 2$ be a positive number greater than each of the numbers 1 , $\left|a_{0}\right|,\left|a_{1}\right|, \cdots,\left|a_{n-1}\right|$. Then it is easy to see that

$$
\left|a_{i}^{(k)}\right|<c^{k+1}
$$

for all $i=0,1,2, \cdots, m-1$, and for all nonnegative integers $k$. Also, the $m$ functions $w, \Delta w, \Delta^{2} w, \cdots, \Delta^{m-1} w$ are each bounded by a positive constant $\lambda$ on the closure of $B(P, r)$. Now, it has already been shown in [3, p. 289] that the remainder term $\int_{B(P, r)} v_{m+k} \Delta^{m+k} w d \rho$ in (16) tends to zero as $k$ tends to $\infty$, provided $w \in C^{2}(R)$ and is a solution of $\Delta w-c w=0$ in $R$, where $c$ is a positive constant. Since $g$ $\left.=\exp (c / n)^{1 / 2} \sum_{i=1}^{n} x_{i}\right)$ is in $C^{2}(R)$ and satisfies $\Delta w-c w=0$ in $R$, where $c$ is given by (19), we have

$$
\lim _{k \rightarrow \infty} \int_{B(P, r)} v_{m+k} \Delta^{m+k} g d \rho=0 .
$$

Let $g_{0}$ be the minimum of $g$ on the closure of $B(P, r)$. Then $g_{0}>0$ and

$$
\begin{aligned}
0 & \leqq c^{m+k} g_{0} \int_{B(P, r)} v_{m+k} d \rho \leqq \int_{B(P, r)} v_{m+k} c^{m+k} g d \rho \\
& =\int_{B(P, r)} v_{m+k} \Delta^{m+k} g d \rho .
\end{aligned}
$$

From (20) and (21) we see that

$$
\lim _{k \rightarrow \infty} c^{m+k} \int_{B(P, r)} v_{m+k} d \rho=0 .
$$

Now, returning to the relation (16), where $w$ satisfies the equation (12), we see that

$$
\begin{aligned}
0 & \leqq\left|\int_{B(P, r)} v_{m+k} \Delta^{m+k} w d \rho\right| \leqq \int_{B(P, r)} v_{m+k}\left|\Delta^{m+k} w\right| d \rho \\
& \leqq\left(m \lambda / c^{m-1}\right) c^{m+k} \int_{B(P, r)} v_{m+k} d \rho .
\end{aligned}
$$

From (22) and (23) it now follows that the remainder term $\int_{B(P, r)} v_{m+k} \Delta^{m+k} w d \rho$ in (16) tends to zero as $k$ tends to $\infty$. Hence, we have 


$$
\frac{1}{\Omega_{r}} \int_{S(P, r)} w d \sigma=\Gamma(n / 2) \sum_{\nu=0}^{\infty}(r / 2)^{2 \nu} \frac{\Delta^{v} w(P)}{\nu ! \Gamma(\nu+n / 2)} .
$$

Similarly,

$$
\frac{1}{\Omega_{r}} \int_{S(P, r)} u w d \sigma=\Gamma(n / 2) \sum_{\nu=0}^{\infty}(r / 2)^{2 \nu} \frac{u(P) \Delta^{v} w(P)}{\nu ! \Gamma(\nu+n / 2)} .
$$

From (24) and (25) we get

$$
\int_{S(P, r)} u w d \sigma=u(P) \int_{S(P, r)} w d \sigma,
$$

for each $S(P, r)$ which together with its interior $B(P, r)$ lies in $R$. This means by Theorem 3 of [1] that $u \in S(w, R)$. This completes the proof.

III. As mentioned in the Introduction, we will now give an example to show that in $E_{n}, n>2, S(w, R)$ can be infinite dimensional even if the W. F. $w$ is not an eigenfunction of the Laplace operator.

In $E_{3}$ let us consider the following W. F.:

$$
w\left(x_{1}, x_{2}, x_{3}\right)=e^{x_{3}}+e^{2 x_{3}}, \quad \text { for all }\left(x_{1}, x_{2}, x_{3}\right) \in E_{3} .
$$

Clearly, $w$ is not an eigenfunction of the Laplace operator. We have

$$
\Delta^{2} w=5 \Delta w-4 w, \quad w_{x_{1}}=w_{x_{2}}=(\Delta w)_{x_{1}}=(\Delta w)_{x_{2}}=0
$$

in $E_{3}$. Hence, by Theorem 2, $S\left(w, E_{3}\right)$ is the solution space of the system of elliptic equations:

$$
\begin{array}{r}
w \Delta u+2 \sum_{i=1}^{3} u_{x_{i}} w_{x_{i}}=0 \\
\Delta w \cdot \Delta u+2 \sum_{i=1}^{3} u_{x_{i}}(\Delta w)_{x_{i}}=0
\end{array}
$$

in $E_{3}$.

Now, for each positive integer $k$, let $P_{k}\left(x_{1}, x_{2}\right)$ be a harmonic polynomial of degree $k$ in $E_{2}$. Then each of the functions

$$
f_{k}\left(x_{1}, x_{2}, x_{3}\right)=P_{k}\left(x_{1}, x_{2}\right), \quad k=1,2,3, \cdots, \infty,
$$

is defined in $E_{3}$ and is harmonic in $E_{3}$. Hence,

$$
\Delta f_{k}=0=\left(f_{k}\right)_{x_{3}}
$$

in $E_{3}$, for all positive integers $k$. It is clear that each of the functions $f_{k}$ is a solution of system (24) of equations. Therefore, $f_{k} \in S\left(w, E_{3}\right)$ 
for each positive integer $k$. Since the infinite set of functions $\left\{f_{1}, f_{2}, \cdots, f_{k}, \cdots\right\}$ is linearly independent over $E_{3}$, it follows that $S\left(w, E_{3}\right)$ is infinite dimensional.

It is also clear that similar types of examples can be constructed in four- and higher-dimensional Euclidean spaces.

\section{BIBLIOGRAPHY}

1. Anil K. Bose, Functions satisfying a weighted average property, Trans. Amer. Math. Soc. 118 (1965), 472-487.

2. - Functions satisfying a weighted average property. II, Trans. Amer. Math. Soc. 124 (1966), 540-551.

3. R. Courant and D. Hilbert, Methods of mathematical physics, Vol. II, Interscience, New York, 1962.

University of Alabama 